\title{
Ataxia telangiectasia: un desorden multisistémico con inestabilidad cromosómica y predisposición al cáncer
}

\author{
Guzmán M. ${ }^{1}$
}

El síndrome de ataxia telangiectasia (A-T) fue descrito por Syllabay Henner en 1926 y redescrito en 1941 por Louis Bar $(1,2)$. Es una entidad autosómica recesiva que afecta a hombres y mujeres en igual proporción. La expresividad del gen A-T es variable y la incidencia familiar es alta $(1,2)$. Se ha estimado que la frecuencia de individuos homocigotos (afectados) para el gen A-T, es de $1 / 40.000$ nacidos vivos y $1 \%$ de la población general serían heterocigotos (portadores). Sin embargo, la incidencia en la población se puede incrementar ya que los homocigotos pueden tener descendencia. Es así como se estima que en la población blanca de Estados Unidos los heterocigotos podrían llegar a constituir el $1,4 \%$ de la población $(2,3)$. Otros autores estiman una frecuencia de heterocigotos mucho más alta entre $0,68 \%$ y $7,7 \%$ de la población (4).

La ataxia telangiectasia es un desorden considerado como multisistémico con diversas manifestaciones clínicas, cuyas características principales son:

1- Ataxia cerebelosa progresiva, telangiectasias especialmente de la conjuntiva, propensión a infecciones sinopulmonares, apraxia oculomotora con inhabilidad en el movimiento voluntario del ojo $(1,2)$.

2- Disfunción inmune variada.

3- Exceso de sensibilidad a la radiación.

4- Inestabilidad cromosómica marcada.

1 Profesora Asistente, Facultad de Medicina, Instituto de Biotecnología, Universidad Nacional, Santafé de Bogotá, Colombia.
5- Alteración de la cinética celular.

6- Alta predisposición al cáncer (3-12).

La inmunodeficiencia consiste en un defecto funcional y numérico de las células $T$ y una deficiencia selectiva de $\lg G 2$, lgG4, $\lg A$, $\lg E$, con un nivel aumentado de IgM (13). Mientras que en individuos normales los timocitos tempranos expresan receptores de cadenas gamma/delta y los linfocitos T maduros expresan receptores de cadenas alfa/beta (14) en 10 pacientes A-T se encontró que hay una proporción aumentada de células $T$ que expresan receptores de cadenas gamma/delta sobre los que expresan receptores alfa/beta (5). Estos hallazgos reflejan un defecto primario que podría interferir con el rearreglo o recombinación somática de los genes de células Ty de las inmunoglobulinas, o con la capacidad para reparar daño en el DNA (15). Sin embargo, Hsieh et al. (16) informan que la señal y la codificación para la formación de la reacción de recombinación $\mathrm{V}$ (D) J es normal en las células A-T.

La respuesta de las células A-T a la radiación ionizante in vitro apoya el postulado de que la base del problema en estos pacientes es un defecto en la reparación del DNA, o un defecto a nivel de la iniciación de la síntesis o, en general, es un defecto en el mecanismo de recombinación del DNA. Los siguientes ejemplos presentan algunas evidencias.

La exposición de los cultivos de linfocitos y de fibroblastos a la radiación ionizante, causa mayor muerte celular de las células A-T comparada con células normales, constituyendo un cuadro de hipersensibilidad a la radiación o radiosensibilidad $(8,17)$. Además, comparada con la respuesta de 
las células normales, la radiación ionizante inhibe más drásticamente la tasa de síntesis del DNA de las células A-T, lo que se conoce como radioresistencia (17). Esto último quiere decir, que las células de individuos normales requieren de un tiempo adicional para la síntesis del DNA con el objeto de reparar el daño causado por la irradiación o por cualquier otro agente mutagénico, en otras palabras, las células normales pueden realizar una reparación del DNA postsíntesis. Sin embargo, las células A-T no se toman el tiempo de reparación postreplicativa, porconsiguiente no pueden reparar el daño del DNA. Lo anterior conduce a la muerte celular o a la producción de micronúcleos y de aberraciones cromosómicas con una mayor frecuencia. También, la exposición de células A-T al compuesto bleomicina conduce a mayor frecuencia de rupturas cromosómicas que lo observado en las células de individuos normales expuestas a este mismo agente clastogénico (18).

Según el grado de radiorresistencia de las células de diferrentes pacientes A-T, se han propuesto cinco grupos de complementación, basados en la tasa de síntesis del DNA. La tasa de síntesis puede variar dentro de un rango del $30 \%$ al $40 \%$ en heterocariones no irradiados. Además, los grupos de complementación ( $A, B, A B, C$ y $D$ ) se han postulado también dependiendo del grado de inhibición de la síntesis de DNA después de irradiación de los heterocariones $(19,20)$.

Más recientemente, se informa otro grupo de complementación adicional con lo cual se ampliaría el espectro de heterogeneidad de la entidad A$T(21)$. Chen et al. (22) informan 8 grupos de complementación en estudios de fusión celular entre células A-T y células de pacientes con el síndrome de Alzheimer o de pacientes con síndrome de Down. Los hallazgos sugieren que estos tres grupos de desórdenes neuronales degenerativos podrían tener en común algún elemento de superposición entre los defectos genéticos.

La información presentada anteriormente sugiere que el defecto de las células A-T puede ser en la iniciación de la síntesis del DNA o debida a un problema específico de la reparación del DNA (23). También se ha propuesto que la base de la anormalidad en A-T es un defecto en la recombinación genética que interfiere con el rearreglo de los genes de las inmunoglobulinas y con la reparación del daño del DNA. El fenómeno de recombinación fue estudiado en líneas de fibroblastos A-T y de controles a través de dos vectores de recombinación. La rata de recombinación intracromosómica fue 30 a 200 veces más alta en fibroblastos A-T que en normales mientras que la recombinación extracromosómica fue similar a lo normal. Por tanto, el aumento en la frecuencia de recombinación es un componente de inestabilidad genética en A-T y puede contribuir con el alto riesgo de cáncer de estos pacientes (24).

Otra manifestación importante en las células de pacientes A-T es la alteración del ciclo celular. Utilizando el método de autorradiografía se pudo establecer que las células linfoblastoides derivadas de pacientes A-T tienen un tiempo total de ciclo celular significativamente más largo, que las células de individuos normales. La diferencia se manifiesta principalmente por el alargamiento de la fase de síntesis (S) (11), lo que conduce a una reducción general de la proliferación celular. Este hallazgo se podría correlacionar con la baja respuesta proliferativa ante el estímulo de lectinas mitogénicas como la fitohemaglutinina de fríjol (PHA). Con relación a la cinética celular, otro estudio muestra que hay discrepancia acerca de si en las células A-T la proteína p53 (gen supresor tumoral TP53) es deficiente o normal. Normalmente la proteína p53 está involucrada en el control de algunos pasos de la cinética celular. En 6 pacientes A-T analizados no se encontró mutación en el gen TP53 lo cual podría sugerir que este gen no está directamente asociado con la susceptibilidad al cáncer de los pacientes A-T (25).

Es muy interesante la demostración realizada al cocultivar plasma o linfocitos de pacientes A-T con el de individuos normales. Se produce un aumento signifivo en el número de aberraciones cromosómicas de las células normales. Es decir, que se ha demostrado un factor clastogénico difusible en el plasma de pacientes A-T. Este factor difusible se detecta también en el medio de cultivos de fibroblastos de piel de estos pacientes (26). La presencia de este factor difusible en 
cultivos in vitro podría utilizarse como un elemento diagnóstico por su efecto clastogénico sobre células normales.

\section{Mapeo genético y cromosomas en A-T}

El gen A-T ha sido mapeado en el brazo largo del cromosoma 11 entre las bandas 22 y 23 (11 q2223). Para el mapeo se utilizó el método del análisis de ligamiento con dos marcadores genéticos THY1 y D11S144 (28). Recientemente, Wei et al. (29) han precisado la localización del gen A-T en la región centromérica del marcador D11S144. Además, McConville et al. (30) caracterizaron otros dos loci polimórficos (D11S535 y D11S611) los cuales permitirán proveer mayor información sobre el locus A-T y podrán ser utilizados como marcadores polimórficos para análisis de ligamiento genético.

Las aberraciones cromosómicas como las traslocaciones, las deleciones y/o las inversiones se pueden producir en cualquier fase del ciclo celular (9). También, se ha observado que las células A-T tienen una diferencia intrínseca en la estructura de la cromatina y se plantea que esta constitución especial pueda estar relacionada con el fenómeno de radiorresistencia de las células A-T (8). Por ejemplo, Almeida et al. (27) informan que las líneas linfoblastoides de células A-T están fuertemente hipometiladas y que los segmentos heterocromáticos de los cromosomas 1 y 16 se encuentran más elongados o descondensados en estas líneas celulares comparado con las células controles.

Aunque el gen A-T se ha mapeado en el cromosoma 11 , la inestabilidad cromosómica de esta entidad (31), no compromete precisamente a este cromosoma sino que los rearreglos espontáneos comprometen a otros cromosomas preferencialmente los cromosomas 7 y 14 . La estadística muestra que estos rearreglos son 25 veces más frecuentes en los pacientes A-T comparado con la frecuencia de rearreglos espontáneos en linfocitos humanos no malignos. Dos sitios específicos de cada cromosoma, son los que están mayormente involucradosenestos rearreglos cromosómicos. En el cromosoma 7 los puntos $7 p 14$ y $7 q 35$ y en el cromosoma 14, los puntos 14q12 y 14qter. Los rearreglos forman inversiones, inv (7) (p14;q35) o inv (14) (q12qter) o también traslocacionest $(7 ; 14)(p 14 ; q 12)$ ot $(7,14)(q 35 ; q 12)$, o la traslocación en tándem $\mathrm{t}(14 ; 14)(6,10,32,33)$. Aunque para varios investigadores las evidencias favorecen que los rearreglos sean de origen monoclonal $(10,34)$, otras evidencias no descartan la posibilidad de que ocurran varias mutaciones en tipos celulares diferentes (pero que produzcan el mismo tipo de rearreglo), condicionando un origen de tipo policlonal (32).

Es importante resaltar que los cuatro sitios de ruptura que comprometen a los cromosomas 7 y 14, también intervienen en la formación de rearreglos con los cromosomas 2 y 22 , en los sitios 2p11, 2p12, 22q12 y 22q13. En los cromosomas 2 y 22 se han mapeado algunos genes para las cadenas de las inmunoglobulinas. Se ha postulado entonces que los rearreglos cromosómicos se favorecerían por la presencia de secuencias de DNA homólogas o parcialmente homólogas con los genes de inmunoglobulinas y del receptor de las células T (6).

No está completamente claro, cuál sería el papel que tienen estos rearreglos cromosómicos en la biología del síndrome A-T. Hay evidencias que sugieren que aquéllos linfocitos que portan una traslocación t $(14 ; 14$ ) (q12;q32) o aquéllos, en los que la traslocación compromete a la región 14q32.1, tienen una ventaja proliferativa que puede resultar en expansión clonal. Inicialmente esta expansión no tendría manifestaciones clínicas, condición que podría perdurar hasta por 10 años. Se requeriría de un evento genético adicional para determinar un fenotipo maligno (33). También, Al Saadi et al. (10) asocian la pérdida de un pequeñomarcador cromosómico 14 (14pter14q12:) con un aumento en la capacidad de transformación maligna, especialmente, hacia tumores linforreticulares.

Ahora, la pregunta que sigue sería ¿cuál es la respuesta de los individuos heterocigotos a la irradiación y a la cinética celular? Se ha informado que las células de estos individuos no muestran una radiorresistencia intermedia, comparada con la respuesta de células A-Ty la de células normales no trasmisoras. Sin embargo, los heterocigotos 
sí muestran una respuesta intermedia cuando se compara la frecuencia de aberraciones cromosómicas producidas porlairradiación X(19). También, los heterocigotos pueden mostrar una mayor frecuencia de aberraciones cromosómicas adquiridas o espontáneas, cuando se comparan estos parámetros en células de la población normal. La respuesta intermedia de las células irradiadas de los individuos heterocigotos se ha tomado como un elemento diagnóstico para detectar individuos trasmisores del gen A-T (34).

\section{Predisposición a cáncer en homocigotos y heterocigotos}

Aproximadamente 1 de 10 pacientes A-T desarrolla cáncer. Esta incidencia es 1.200 veces mayor que aquélla observada en la población control según la edad (35). La primera causa de muerte en estos pacientes es la enfermedad puimonar crónica progresiva (83\%) y la segunda el cáncer $(50 \%)$. Prevalecen los linfomas no Hodking $(41 \%)$ y las leucemias $(23 \%)$ que en conjunto constituyen un $64 \%$, seguidos de tumores sólidos (26\%) especialmente de la cavidad oral, seno, estómago, páncreas, ovario e hígado (12). La alta prevalencia de linfomas y leucemia en pacientes A-T constituye un grave problema para el tratamiento, puesto que como se ha venido mencionando, estos pacientes tienen una fuerte hipersensibilidad a la radiación lo que ha sugerido, por su parte, un defecto en el mecanismo de reparación del DNA $(8,9,17)$.

Sin embargo, la predisposición al cáncer no es exclusiva de los individuos homocigotos sino que es evidente también en los individuos heterocigotos. Los heterocigotos para el gen A-T, menores de 45 años tienen un riesgo 5 veces mayor de desarrollar cáncer que la población general según la edad (3). El riesgo de cáncer de seno para una heterocigota $A$-T es 6,8 veces mayor que el de la población control y se estima que en los E.U.A., del $9 \%$ al $18 \%$ de todos los cánceres de seno deben ser aportados por heterocigotas A-T. El gen A-T predispone a losheterocigotos ai desarrollo de ciertos tipos de cáncer, especialmente a leucemias, linfomas y carcinomas de ovario, seno, gástrico, cervical, de colon y de vías biliares (5).
Sin embargo, en un estudio reciente Wooster et al. (36) analizaron 16 familias con cáncer de seno a través de 5 marcadores en el cromosoma 11q. Los autores no encontraron ninguna evidencia para establecer ligamiento entre el cáncer de seno y los marcadores en el cromosoma 11. Según los autores, la contribución de heterocigotos para el cáncer de seno es mínima.

Finalmente, se puede concluir que las diversas manifestaciones clínicas de esta entidad son interdependientes y la interdependencia puede focalizarse como defecto de la reparación del DNA (35) o como un defecto general de recombinación genética $(24,37)$ que conduce a inestabilidad cromosómica (38-40) y al desarrollo de cáncer (33). En la actualidad, se puede considerar al síndrome de ataxia telangiectasia como un modelo de una enfermedad genética que predispone al cáncer $(12,28,29,39)$, que permite estudiar diferentes problemas del desarrollo biológico normal y anormal.

\section{Referencias}

1. Mckusick V. Mendelian inheritance in man. The Johns Baltimore: Hopkins University Press. 7 th. 1986; 848.

2. Swift M, Sholman L, Perry M, et al. Malignant neoplasms in the families of patients with ataxia telangiectasia. Cancer Res 1976; 36:209.

3. Swift M, Chase Ch, Morrell D. Cancer predisposition of ataxia telangiectasia heterocygotes. Cancer Genêt Cytogent 1990; 46:21.

4. Murnane J, Kapp L. A critical look at the association of human genetic syndromes with sensibility to ionizing radiation. Semin Cancer Biol 1993; 4:93.

5. Carbonari M, Cherchi M, Paganelli R, et al. Relative increase of $T$ cells expressing the gamma/delta rather than the alpha/beta receptor in ataxia telangiectasia. Eng J Med 1990; 322:73.

6. Aurias A, Dutriliaux B. Probable involvement of immunoglobulin superfamily genes in most recurrent chromosomal rearrangements from ataxia telangiectasia. Hum Genet 1986; 72:210.

7. Smith P, Paterson M. Defective DNA repair and increase lethality in ataxia telangiectasia cells exposed to 4nitroquinoline-1-oxide. Nature 1980; 287:747.

8. Painter R. Structural changes in chromatin as the basis for radiosensitivity in ataxia telangiectasia. Cytogenet Cell Genet 1982; 33:139.

9. Natarajan A, Meyers M. Chromosomal radiosensitivity of ataxia telangiectasia cells at different cell cycle stages. Hum Genet 1979; 52:127.

10. Al Saadi A, Palutke M, Kumar K. Evolution of chromosomal abnormalities in sequential cytogenetic studies of ataxia telangiectasia. Hum Genet 1980; 55:23. 
11. Cohen M, Simpson S. Growth kinetic of ataxia telangiectasia lymphoblastoid cells. Evidence for a prolonged S period. Cytogenet Cell Genet 1980; 28:24.

12. HechtF, Hecht B. Cancer in ataxia telangiectasia patients. Cancer Genet Cytogenet 1990; 46:9.

13. Fiorilli M, Businco L, Randolfi F. Heterogeneity of immunological abnormalities in ataxia telangiectasia. $J$ Clin Immunol 1983; 3:135.

14. Allison J, Lanier L. Structure, function, and serology of the T-cell antigen receptor complex. Ann Erv Immuno: $1987 ; 5: 503$.

15. Fiorilli M, Carbonari M, Crescemzi M. T-celi receptor genes and ataxia telangiectasia. Nature 1985; 313:186.

16. Hsieh C, Arlett C, Lieber M. V (D) J recombination in ataxia telangiectasia, Bloom's syndrome, and a DNA ligase I associated immunodeficiency disorder. J Biol Chem 1993; 268:20105.

17. Painter R, Young B. Radiosensivity in ataxia telangiectasia: a new explanation. Proc Natl Acad Sci USA 1980; 77:7.315.

18. Leuzzi V, Elli R, Antonelli A, et al. Neurological and cytogenetic study in early-onset ataxia telangiectasia patients. Eur J Pediat 1993; 152:609.

19. Natarajan A, Meijers M, Van Zeeland A, et al. Attempts to detect ataxia telangiectasia (A-T) heterzygotes by cytogenetical techniques. Cytogenet Cell Genet 1982; 33:145.

20. Chen P, Kidson C. Complementation analysis in ataxia telangiectasia: fibroblast-lymphoblastoid cell heterokaryon assay by radiation-induced chromosome aberrations. Cytogenet Cell Genet 1993; 64:9.

21. Hernádez D, McConville C, Stacey $\mathrm{M}$, et al. A family showing no evidence of linkage between the ataxia telangiectasia gene and chromosome 11q22-23. J Med Genet 1993; 30:135.

22. Chen P, Kidson C, Lavin M. Evidence of different complementation groups among human genetic disorders characterized by radiosensivity.

23. Murnane J, Painter R. Complementation of the defects in DNA synthesis in irradiated and un-irradiated ataxia telangiectasia cells. Proc Natl Acad Sci USA 1982; 79:1960.

24. Jonveaux P, Berger R. Lack of mutations in the p53 gene exons 5 to 8 in ataxia telangiectasia. Cancer Genet Cytogenet 1993; 66:128.

25. Meyn M. High spontaneous intrachromosomal recombination rates in ataxia telangiectasia. Science 1993; 260:1327.
26. Shaham M, Becker Y, Cohen M. A diffusable clastogenic factor in ataxia telangiectasia. Cytogenet Cell Genet 1980; 27:155

27. Almeida A, Kokalj Vokac N, Lefrancois D, et al. Hypomethylation of classical satellite DNA and chromosome instability in lymphoblastoid cell lines. Hum Genet 1993. 91: 538.

28. Gatti R, Berkel I, Boder E, et al. Localization of an ataxia telangiectasia gene to chromosome 11q22-23 Nature 1988; 336:577.

29. Wei S, Rocchi M, Archidiacono N. Physical mapping of the human chromosome $11 \mathrm{q} 23$ region containing the ataxia telangiectasia locus. Cancer Genet Cytogenet 1990; $46: 1$

30. McConvilie C, Byrd P, Ambrose $\mathrm{H}$, et al. Paired STSs amplified from radiation hybrids, and from associated YACs, identify highly polymorphic loci flanking the ataxia telangiectasia locus on chromosome 11q22-23. Hum Mol Genet 1993: 2:969.

31. Hetch F, McCaw B, Koler R. Ataxia telangiectasia clonal growth of transiocation lymphocytes. N Engl J Med 1973; 289:286.

32. Aurias A, Dutrillaux B, Griscelli C. Tandem translocation $t(14 ; 14)$ in isolated and clonal cells in ataxia telangiectasia are different. Hum Genet 1983; 63:320.

33. Russo G, Isobe M, Gatti R. Molecular analysis of a $t(14 ; 14)$ translocation in leukemic T-cells of an ataxia telangiectasia patient. Proc Natl Acad Sci USA 1989; 86:602.

34. Aurias A. Analyse cytogenetique de 21 cas d'ataxie telangiectasie. J Génét Hum 1981; 29:235.

35. Huang P, Sheridan III R. Genetic and biochemical studies with ataxia telangiectasia. A review. Hum Genet 1981; 59:1.

36. Wooster R, Ford D, Mangion J, et al. Absence of linkage to the ataxia telangiectasia locus in familial breast cancer. Hum Gent 1993; 92:91.

37. Peterson R, Funkhouse J. Speculations on ataxia telangiectasia:defective regulation of the immunoglobuline gene superfamily. Immonolology Today 1989; 10:313.

38. Aurias A, Dutrillaux B, Buriot D, et al. High frequencies of inversions and translocations of chromosomes 7 and 14 in ataxia telangiectasia. Mutat Res 1980; 69:369.

39. Hecht F. Hematologic malignancy breakpoint update. Appendice C. Cancer Genet Cytogenet 1988; 31:132.

40. Caporossi D, Tedeschi B, Vernole P. Chromosome breakage induced by bleomycin in an ataxia telangiectasia lymphoblastoid line: correlation with fragile sites and Epstein-Barr virus DNA localization. Cytogenet Cell Genet 1989; 52:180. 